\title{
Reconstrução de região infraorbitária após ressecção de displasia fibrosa com enxerto ósseo bovino liofilizado
}

Reconstruction of the infraorbital region after resection of fibrous dysplasia with lyophilized bovine bone graft

Reconstrucción de la región infraorbitaria tras resección de displasia fibrosa con injerto óseo bovino liofilizado

Alexandre Silva de Quevedo

ORCID: https://orcid.org/0000-0001-5613-8015 Universidade Federal do Rio Grande do Sul, Brasil

E-mail: quevedoalexandre@ hotmail.com

Adriana Corsetti

ORCID: https://orcid.org/0000-0002-0136-1321

Hospital de Clínicas de Porto Alegre, Brasil Universidade Federal do Rio Grande do Sul, Brasil E-mail: adri.corsetti@gmail.com

Angelo Luiz Freddo

ORCID: https://orcid.org/0000-0001-6361-6572

Hospital de Clínicas de Porto Alegre, Brasil

Universidade Federal do Rio Grande do Sul, Brasil E-mail: angelofreddo@gmail.com

Renan Cavalheiro Langie

ORCID: https://orcid.org/0000-0001-6249-2862 Complexo Hospitalar Santa Casa de Misericórdia de Porto Alegre, Brasil E-mail: renanlangie@gmail.com
Deise Ponzoni

ORCID: https://orcid.org/0000-0003-2855-7495

Hospital de Clínicas de Porto Alegre, Brasil Universidade Federal do Rio Grande do Sul, Brasil

E-mail: deise.ponzoni@ufrgs.br

\begin{abstract}
Resumo
Introdução: A displasia fibrosa monostótica envolvendo a região maxilo-facial é mais frequente na maxila, podendo ocorrer em outros ossos. Na maioria dos casos, a condição é assintomática e tem um crescimento lento, com evolução autolimitada, associada à maturação esquelética. O tratamento cirúrgico conservador é a conduta de eleição, sempre que possível. Objetivo e relato do caso: $\mathrm{O}$ artigo tem por objetivo apresentar o relato de um caso clínico de paciente jovem, com diagnóstico de displasia fibrosa monostótica zigomática-maxilar. O tratamento proposto foi a ressecção da lesão e reconstrução imediata do defeito com enxerto ósseo bovino liofilizado. $O$ enxerto foi mantido com microplaca e parafusos de titânio. O paciente permaneceu em controle clínico e imaginológico sem evidências de recidiva no período pós-operatório de seis anos. Considerações finais: Na reconstrução imediata, o enxerto ósseo bovino liofilizado atuou como arcabouço osteocondutor e favoreceu a recuperação volumétrica da área, proporcionando suporte para reorganização do defeito ósseo.
\end{abstract}

Palavras-chave: Enxerto ósseo; Materiais biocompatíveis; Cirurgia bucal.

\section{Abstract}

Introduction: Monostotic fibrous dysplasia involving the maxillofacial region is more frequent in the maxilla and may occur in other bones. In most cases, the condition is asymptomatic and has a slow growth, with self-limited evolution, 
associated with skeletal maturation. Conservative surgical treatment is the preferred approach, whenever possible. Objective and case report: The article aims to present the report of a clinical case of a young patient diagnosed with zygomatic-maxillary monostotic fibrous dysplasia. The proposed treatment was the resection of the lesion and immediate reconstruction of the defect with a lyophilized bovine bone graft. The graft was maintained with a titanium microplate and screws. The patient remained under clinical and imaging control with no evidence of recurrence in the six-year postoperative follow-up. Final considerations: In the immediate reconstruction, the lyophilized bovine bone graft acted as an osteoconductive framework and favored the volumetric recovery of the area providing support for the reorganization of the bone defect.

Keywords: Bone transplantation; Biocompatible materials; Surgery oral.

\section{Resumen}

Introducción: La displasia fibrosa monostótica que afecta a la región maxilofacial es más frecuente en el maxilar y puede ocurrir en otros huesos. En la mayoría de los casos, la condición es asintomática y tiene un crecimiento lento, con evolución autolimitada, asociada a la maduración esquelética. El tratamiento quirúrgico conservador es el enfoque preferido, siempre que sea posible. Objetivo y reporte de caso: El artículo tiene como objetivo presentar el reporte de un caso clínico de un paciente joven diagnosticado de displasia fibrosa monostótica cigomático-maxilar. El tratamiento propuesto fue la resección de la lesión y la reconstrucción inmediata del defecto con injerto óseo bovino liofilizado. El injerto se mantuvo con una microplaca de titanio y tornillos. El paciente permaneció bajo control clínico y de imagen sin evidencia de recidiva en el postoperatorio de seis años. Consideraciones finales: En la reconstrucción inmediata, el injerto óseo bovino liofilizado actuó como marco osteoconductor y favoreció la recuperación volumétrica de la zona proporcionando apoyo para la reorganización del defecto óseo.

Palabras clave: Trasplante óseo; Materiales biocompatibles; Cirugía bucal.

\section{Introdução}

A displasia fibrosa (DF) é uma anomalia do desenvolvimento esquelético caracterizada pela substituição do osso normal pelo excesso de proliferação de tecido fibroso em trabéculas ósseas irregulares (Assiri, 2020; Pereira et al., 2019). Quando envolve apenas um osso é dita monostótica e poliostótica quando aleatoriamente atinge múltiplos ossos ou está associada a manifestações extra-esqueléticas como na Síndrome de McCune-Albrigt (Boyce \& Collins, 2020; HagelsteinRotman et al., 2021; Hartley et al., 2019; Pereira et al., 2019; Pontes-Madruga et al., 2020).

Na região craniofacial, o complexo zigomático-maxilar e o mais afetado pela DF monostótica (Assiri, 2020; Bertin et al., 2020; Pontes-Madruga et al., 2020; Verma et al., 2013). Clinicamente, uma DF causa expansão óssea, sendo geralmente diagnosticada na infância, adolescência ou adulto até terceira década de vida. O início desta condição está associado ao crescimento esquelético ativo podendo apresentar curso autolimitado. Mutações somáticas pós-zigóticas são observadas no gene GNAS1 e associadas à displasia fibrosa (Ahn et al., 2018; Boyce \& Collins, 2020; Pereira et al., 2019). A transformação maligna espontânea da DF é considerada um evento raro (Assiri, 2020; Lee et al., 2012; Sadeghi \& Hosseini, 2011).

A indicação do tratamento cirúrgico, por motivos funcionais, diagnósticos e estéticos, leva em consideração a progressão e a possibilidade de recorrência (Mici \& Belli, 2018). Lesões monostóticas podem ser tratadas através de forma conservadora (cosmética) ou por ressecção cirúrgica associada à reconstrução imediata. O enxerto ósseo bovino liofilizado é biocompatível, de fácil obtenção, alta disponibilidade, e guarda grande semelhança ao osso humano. Permite modelagem da área a ser reconstruída, servindo como arcabouço osteocondutor (Galia et al., 2008; Galia et al., 2011; Galia et al., 2012; Ribeiro et al., 2015).

\section{Metodologia}

Trata-se de estudo retrospectivo, descritivo e observacional no formado de Relato de Caso Clínico (Alsaywid \& Abdulhaq, 2019; Estrela, 2018) de um paciente tratado, sob anestesia geral, em ambiente hospitalar. Os dados descritivos foram coletados através de acesso ao prontuário físico e eletrônico, após autorização do paciente, mediante assinatura do Termo de Consentimento Livre e Esclarecido. Foram feitas buscas na literatura utilizando o MeSH Database através da padronização das palavras-chave. A discussão foi baseada nas publicações anteriores acerca do tema. 


\section{Relato de Caso}

Paciente masculino, 15 anos, hígido, sem histórico de doenças prévias ou alergias, foi encaminhado pela unidade básica de saúde, para avaliação de aumento de volume em face, com evolução de mais de um ano. O paciente relatou ausência de sintomatologias associadas. No exame clínico extra-bucal observou-se aumento de volume limitado e endurecido, em região infraorbitária esquerda. Não foram identificadas alterações nos tecidos moles (Figura 1A).

Na oroscopia foram observadas ausências clínicas dos terceiros molares. Demais tecidos e estruturas intra e peribucais sem alterações. O exame tomográfico de face revelou lesão insuflante, centrífuga, com cerca de $1 \mathrm{~cm}$ de espessura na estrutura óssea medular a partir da porção medial do rebordo infraorbitário esquerdo invadindo soalho da órbita, sugestiva de displasia fibrosa (Figura 1C). Foi registrada a presença de terceiros molares retidos. O exame cintilográfico de esqueleto mostrou captação do radiofármaco na região da lesão.

O paciente foi submetido à intervenção cirúrgica sob anestesia geral para remoção da lesão e reconstrução do defeito. A incisão, em pele, foi realizada na região infraorbitária esquerda. Após divulsão, descolamento e exposição da área afetada, a demarcação limítrofe foi realizada com brocas esféricas, seguida por ostectomia em bloco, conduzida por cinzel e martelo (Figuras 2A e 2C). A reconstrução imediata do defeito foi realizada com enxerto ósseo bovino liofilizado (Figura 2D). Este foi esculpido e adaptado à região, mimetizando o soalho e reborda ósseos, sendo imobilizado com 1 microplaca de 8 furos e 6 parafusos de titânio (Figura 2B). As suturas, organizadas nos planos teciduais, foram finalizadas com o fechamento intradérmico da pele. Na sequência a remoção cirúrgica dos terceiros molares retidos completou o atendimento. A remoção da sutura em pele, ocorreu no décimo dia pós-operatório. O exame histopatológico, confirmou o diagnóstico de displasia. O paciente foi acompanhado através de exames clínicos e tomográficos, não sendo observadas alterações e recidivas no período de controle de seis anos pós-operatórios (Figuras 1B, 1D, 3A e 3B). 
Research, Society and Development, v. 10, n. 8, e14910817295, 2021

(CC BY 4.0) | ISSN 2525-3409 | DOI: http://dx.doi.org/10.33448/rsd-v10i8.17295

Figura 1: Aspectos clínico e tomográfico no pré e pós-operatório do paciente.
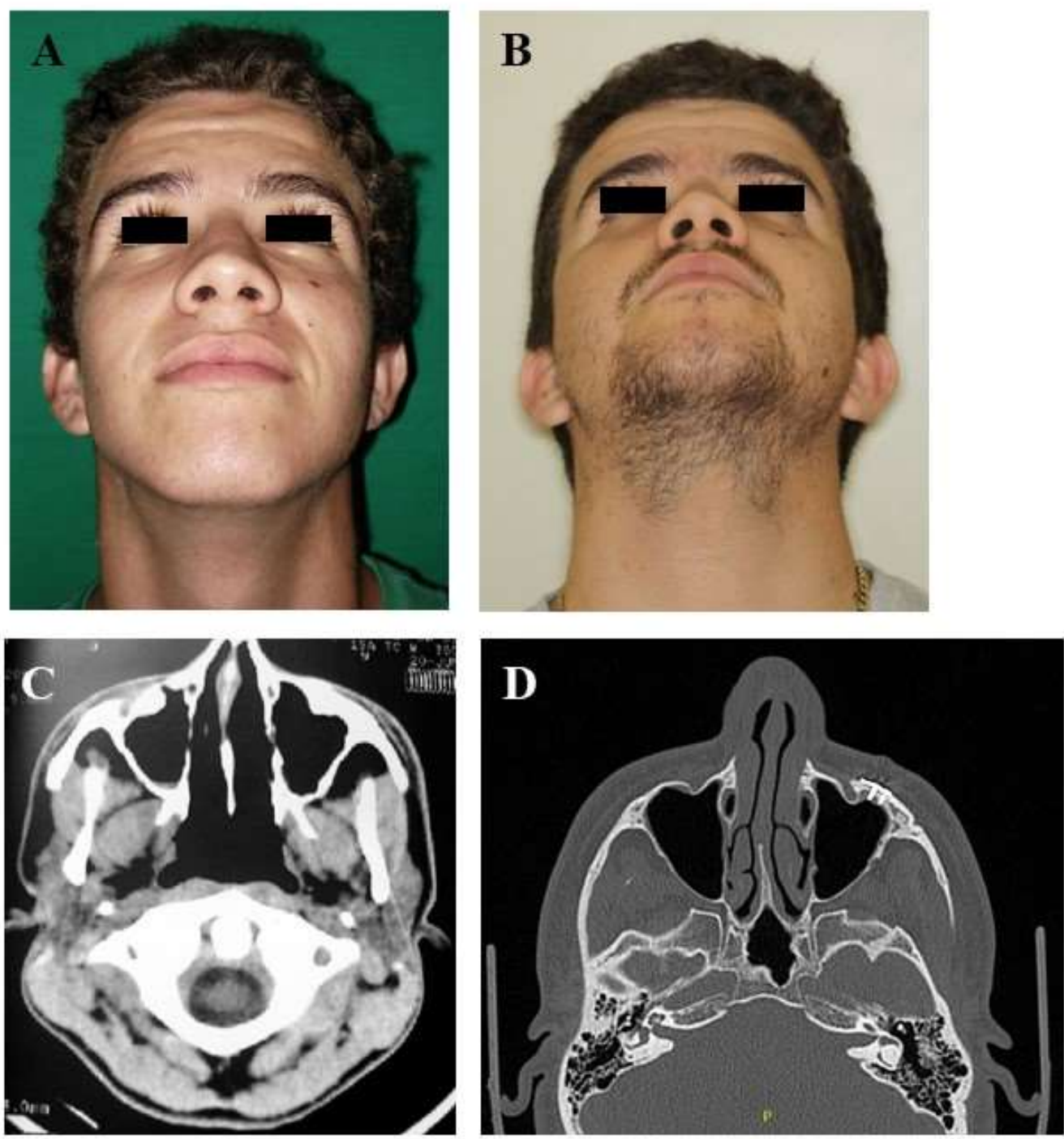

A - Aspecto clínico extra-bucal pré-operatório. Evidencia-se o aumento de volume na região infraorbitária esquerda.

$\mathrm{B}$ - Controle clínico extra-bucal pós-operatório (seis anos). Observa-se asimetria facial e discreta cicatriz em pele associada à região infraorbitária esquerda.

C - Tomografia computadorizada de face pré-operatória, corte axial. Identifica-se lesão insuflante, na estrutura óssea medular da porção medial do rebordo infraorbitário esquerdo.

D - Tomografia computadorizada de face pós-operatória (seis anos), corte axial. Observa-se a integração dos meios de fixação rígida. Não há evidência de sinais que indiquem a recidiva da lesão.

Fonte: Autores.

$\mathrm{Na}$ imagem clínica pré-operatória destaca-se o aumento de volume na região infraorbitária esquerda. O mesmo pode ser observado na imagem tomográfica pré-operatória. Na imagem clínica pós-operatória verifica-se o desenvolvimento e manutenção da simetria facial. A tomografia computadorizada de face, de controle pós-operatório não evidencia sinais de recidiva e mostra a estabilidade dos meios de fixação. 
Research, Society and Development, v. 10, n. 8, e14910817295, 2021

(CC BY 4.0) | ISSN 2525-3409 | DOI: http://dx.doi.org/10.33448/rsd-v10i8.17295

Figura 2: Fases da intervenção cirúrgica na região infraorbitária esquerda, realizada sob anestesia geral.
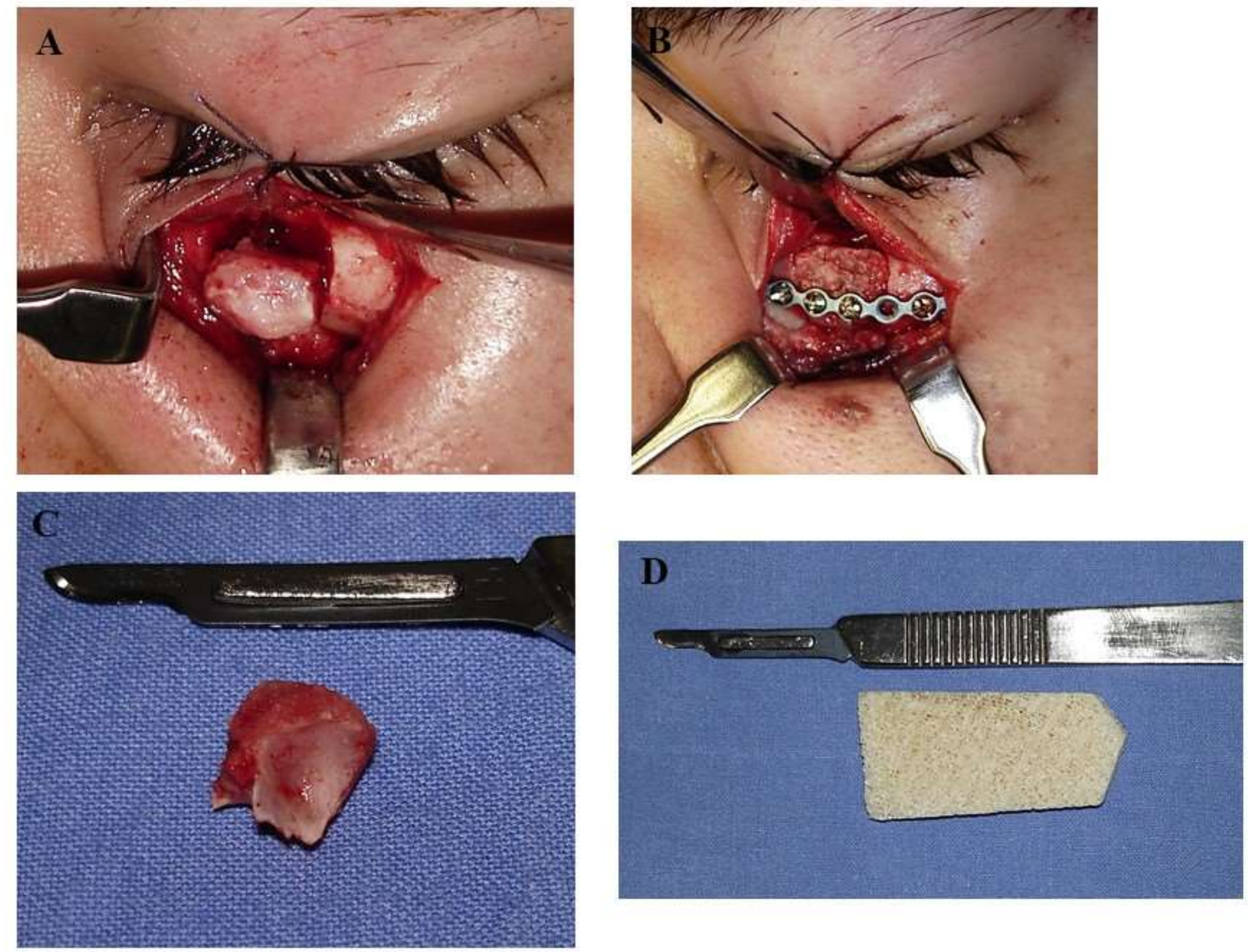

A - Aspecto trans-cirúrgico. Ressecção da área da lesão.

B - Aspecto trans-cirúrgico. Reconstrução imediata com o enxerto ósseo liofilizado bovino e imobilização com microplaca e microparafusos.

C - Peça cirúrgica. Observa-se a borda infraorbitária e a delgada parede do soalho da cavidade orbitária

D - Enxerto ósseo liofilizado bovino. Observa-se a macroestrutura.

Fonte: Autores.

Destaca-se a perfeita adaptação do enxerto ósseo liofilizado bovino na área reconstruída, mantido pelo sistema de microplaca e microparafuros. 
Figura 3: Controle clínico e tomográfico tardios do paciente.
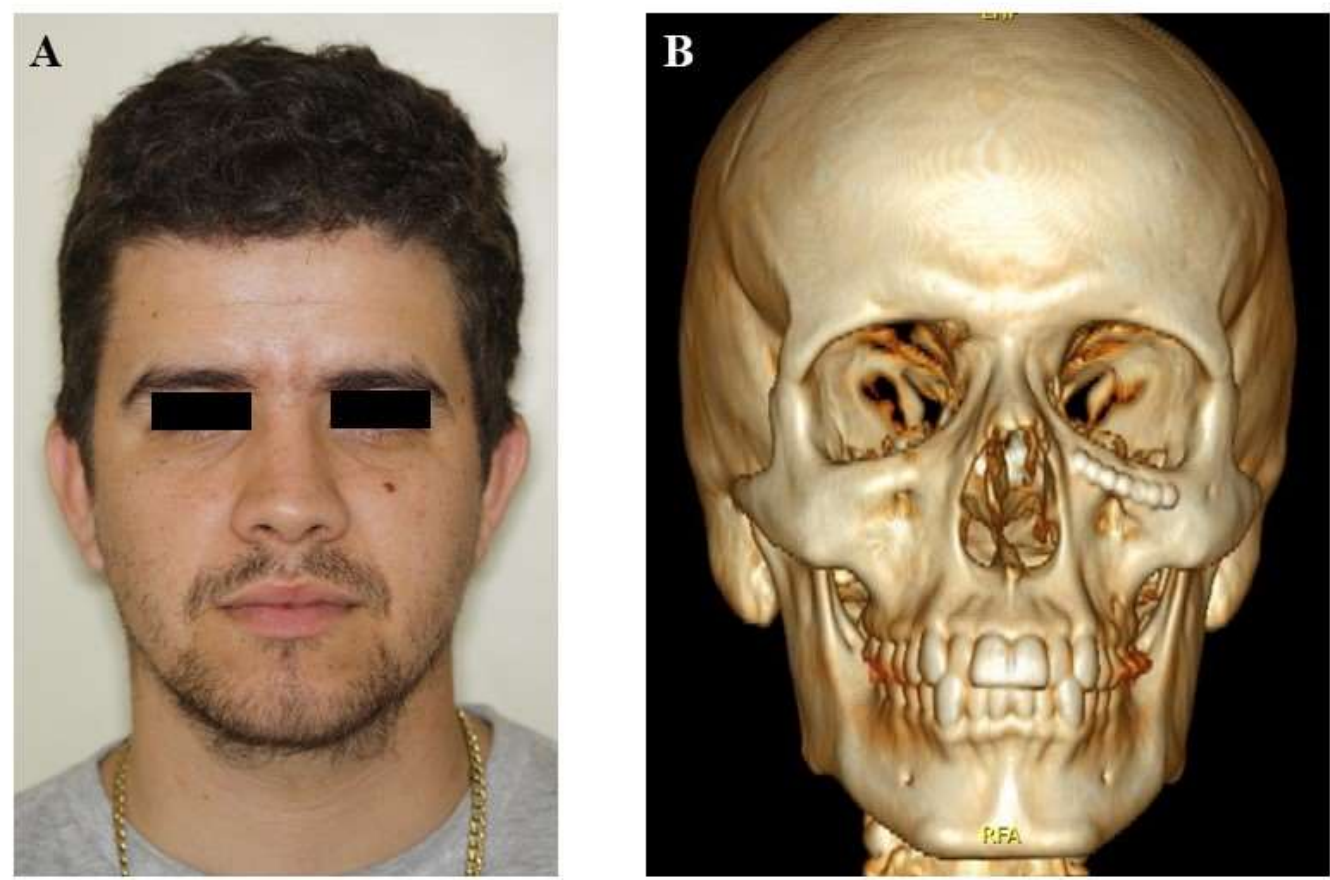

A - Aspecto clínico frontal pós-operatório (seis anos).

B -Tomografia computadorizada de face pós-operatória (seis anos), reconstrução tridimensional. Verifica-se a perfeita adaptação dos meios de fixação rígida, simetria facial e ausência de sinais de recidiva da lesão

Fonte: Autores. operatório tardio.

Observa-se o desenvolvimento simétrico na área operada e estrutura do esqueleto facial, do paciente no controle pós-

\section{Discussão}

O complexo zigomático-maxilar é a região mais envolvida com a DF monostótica (Ahn et al., 2018). O amplo espectro clínico pode variar de lesões solitárias menores, evoluindo para graves alterações esqueléticas com obstrução nasal, maloclusão dentária, dor, comprometendo a visão e audição (Hartley et al., 2019). Esta lesão pode cruzar as suturas e entrar nos ossos adjacentes (Gupta, Garg \& Mittal, 2017), o que explicaria sua expansão no complexo zigomático-maxilar.

O diagnóstico torna-se mais difícil quando a lesão tem evidências clínicas desprezíveis (Gupta, Garg \& Mittal, 2017). Como a maioria dos casos relatados na literatura, também neste, o crescimento foi lento e assintomático (Hwang et al., 2020; Pereira et al., 2019). Na TC não foram observadas áreas osteolíticas, reação periostal contígua à lesão, defeitos corticais ou crescimentos de tecido mole associado, o que indicaria a possibilidade de malignidade. A transformação maligna da DF monostótica craniofacial é considerada um evento raro (menos de 1\%) (Assiri, 2020; Lee et al., 2012; Sadeghi \& Hosseini, 2011;). Os osteossarcomas de baixo grau podem ter a indicação de biópsia, inclusive em pacientes pediátricos (Lee et al., 2012; Valentini et al., 2017). O componente histológico caracterizado por trabéculas alongadas de tecido ósseo com curvas irregulares, dando origem ao padrão de caracteres chineses confirmou uma DF. Para o paciente não foi realizado o exame 
molecular. Sabe-se que mesmo que as mutações de GNAS1 representem um potencial biomarcador para o diagnóstico de displasia fibrosa, sua frequência varia dependendo do método de deteç̧ão molecular usado. Apesar de ocorrer com menor frequência na forma monostótica, nenhum tipo de endocrinopatia foi identificado no paciente (Pereira et al., 2019).

O tratamento da displasia fibrosa continua sendo cirúrgico uma vez excluída a observação clínica (Ahn et al., 2018; Lee et al., 2012). A proposta foi a ressecção, com biópsia total, e reconstrução imediata baseada na localização, características da lesão e possibilidade de reconstrução conservadora, com baixa morbidade ao paciente.

O enxerto autógeno cortical da calvária é um método confiável, com baixas taxas de complicações para a região (Ahn et al., 2018). Considerando a morbidade na área doadora, sua indicação foi descartada para este caso. A possibilidade de utilização do enxerto ósseo bovino liofilizado para reconstrução na região maxilofacial já foi investigada em modelos animais e em humanos (Al Qabbani et al., 2018; Soares et al., 2019; Trotta et al., 2014; Zanettini et al., 2018). No relato de caso foi utilizado o enxerto ósseo liofilizado bovino desenvolvido por Galia (2008), cuja obtenção está baseada no protocolo de Kakiuchi et al. (1996). O enxerto ósseo bovino é tratado quimicamente, liofilizado e esterilizado com radiação gama. A estrutura óssea trabecular e a composição orgânica consistindo em colágeno do tipo 1 e hidroxiapatita são preservadas (Galia et al., 2008; Galia et al., 2011). A liofilização diminui a antigenicidade alterando minimamente a composição físico-química do enxerto quando comparado ao enxerto ósseo não processado (Galia et al., 2011). Não foram relatadas reações imunogênicas ou toxicidade (Trotta et al., 2014). O comportamento histológico do enxerto ósseo bovino liofilizado foi avaliado em humanos, em cirurgias ortopédicas mostrando excelente osteocondutividade biocompatibilidade (Diesel et al., 2017; Galia et al., 2012; Ribeiro et al., 2015,). No caso relatado, os controles tomográficos demonstraram a remodelação progressiva da área com a manutenção da arquitetura óssea e incorporação do enxerto ósseo, confirmados clinicamente pela ausência de rejeição/infecção.

A microplaca e os parafusos que imobilizaram o enxerto ósseo liofilizado bovino não foram removidos no controle pós-operatório. O paciente apresentou osseointegração associada à interface osso-implante sem sintomatologia associada aos aparatos metálicos na região do terço fixo da face, livre de interferência muscular. As evidências de alta insolubilidade do titânio aos fluídos orgânicos, associadas à inexistência da correlação entre o tempo de fixação e o desprendimento de partículas metálicas destacam a capacidade bioinerte e biocompatível do titânio e contraindicam a remoção sem sintomatologia da fixação interna rígida (Acero et al., 1999; Langford \& Frame, 2002; Meningaud et al., 2001; Pan \& Patil, 2014).

\section{Considerações Finais}

A abordagem realizada por equipe cirúrgica experiente foi baseada na extensão da lesão, possibilidade de biópsia total, acesso favorável, sem comprometimento estético e funcional, respeitando a preferência do paciente. $\mathrm{O}$ enxerto ósseo bovino liofilizado é uma alternativa para reconstrução após resseção de lesões, como a displasia fibrosa, envolvendo as estruturas maxilo-faciais. Esse tipo de enxerto é biocompatível e osteocondutor, apresentando características físico-químicas que proporcionam uma estrutura temporal de suporte para reorganização dos defeitos ósseos. O enxerto ósseo autógeno, por sua morbidade, pode não ser recomendado em pequenas ou médias reconstruções, como neste caso. Nenhuma sequela foi associada ao paciente, possivelmente pelo estabelecimento do diagnóstico e tratamento em fase precoce de desenvolvimento.

O enxerto ósseo liofilizado bovino apresenta alta disponibilidade e pode ser indicado para reconstrução de defeitos ósseos na Odontologia e na Ortopedia e Traumatologia. Novos estudos clínicos, com número amostral maior, devem ser incentivados, uma vez que sua aplicação é segura e diminui a morbidade dos pacientes, por dispensar um sítio doador. 


\section{Referências}

Acero, J., Calderon, J., Salmeron, J. I., Verdaguer, J. J., Concejo, C., \& Somacarrera, M. L. (1999). The behavior of titanium as a biomaterial: microscopy study of plates and surrounding tissues in facial osteosynthesis. Journal of cranio-maxillo-facial surgery: official publication of the European Association for Cranio-Maxillo-Facial Surgery, 27(2), 117-123.

Ahn, S. J., Hong, J. W., Kim, Y. O., Lew, D. H., \& Lee, W. J. (2018). Treatment of fibrous dysplasia of the zygomatico maxillary complex with radical resection and three-dimensional reconstruction with autologous calvarial bone graft. Archives of craniofacial surgery, 19(3), 200-204.

Al Qabbani, A., Al Kawas, S., A Razak, N. H., Al Bayatti, S. W., Enezei, H. H., Samsudin, A. R., \& Sheikh Ab Hamid, S. (2018). Three-dimensional radiological assessment of alveolar bone volume preservation using bovine bone xenograft. The Journal of craniofacial surgery, 29 (2), e203-e209.

Alsaywid, B. S., \& Abdulhaq, N. M. (2019). Guideline on writing a case report. Urology annals, 11(2), $126-131$.

Assiri, K. I. (2020). Monostotic fibrous dysplasia involving the mandible: A Case Report. SAGE open medical case reports, 8, $2050313 X 20936954$.

Bertin, H., Huon, J. F., Guillot, P., Longis, J., Corre, P., Bordereau, S., \& Lebranchu, P. (2020). Fibrous dysplasia of the orbital region: Series of 12 cases and review of the literature. Journal francais d'ophtalmologie, 43(6), 467-476.

Boyce, A. M., \& Collins, M. T. (2020). Fibrous dysplasia/McCune-Albright Syndrome: A rare, mosaic disease of Gas activation. Endocrine reviews, 41(2), $345-370$.

Diesel, C. V., Ribeiro, T. A., Guimarães, M. R., Macedo, C., \& Galia, C. R. (2017). Acetabular revision in total hip arthroplasty with tantalum augmentation and lyophilized bovine xenograft. Revista brasileira de ortopedia, 52(Suppl 1), 46-51.

Estrela, C. (2018). Metodologia científica: Ciência, ensino e pesquisa. Arte Med.

Galia, C. R., De Luca, G., Jr, Ávila, L. M., Rosito, R., \& Macedo, C. A. (2012). Bovine lyophilized graft (blg): histological analysis on behavior in humans after 49 months. Revista brasileira de ortopedia, 47(6), 770-775.

Galia, C. R., Lourenço, A. L., Rosito, R., Souza Macedo, C. A., \& Camargo, L. M. (2011). Physicochemical characterization of lyophilized bovine bone grafts. Revista brasileira de ortopedia, 46(4), 444-451.

Galia, C. R., Macedo, C. A., Rosito, R., Mello, T. M., Camargo, L. M., \& Moreira, L. F. (2008). In vitro and in vivo evaluation of lyophilized bovine bone biocompatibility. Clinics (Sao Paulo, Brazil), 63(6), 801-806.

Gupta, D., Garg, P., \& Mittal, A. (2017). Computed tomography in craniofacial fibrous dysplasia: A case series with review of literature and classification update. The open dentistry journal, 11, 384-403.

Hagelstein-Rotman, M., Meier, M. E., Majoor, B., Cleven, A., Dijkstra, P., Hamdy, N., van de Sande, M., Dekkers, O. M., \& Appelman-Dijkstra, N. M. (2021). Increased prevalence of malignancies in fibrous dysplasia/McCune-Albright Syndrome (FD/MAS): data from a National Referral Center and the Dutch National Pathology Registry (PALGA). Calcified tissue international, 108(3), 346-353.

Hartley, I., Zhadina, M., Collins, M. T., \& Boyce, A. M. (2019). Fibrous dysplasia of bone and McCune-Albright Syndrome: A bench to bedside review. Calcified tissue international, 104(5), 517-529.

Hwang, D., Jeon, J., Hong, S. H., Yoo, H. J., Choi, J. Y., \& Chae, H. D. (2020). Radiographic follow-up of fibrous dysplasia in 138 patients. AJR. American journal of roentgenology, 215(6), 1430-1435.

Kakiuchi, M., Ono, K., Nishimura, A., \& Shiokawa, H. (1996). Preparation of bank bone using defatting, freeze-drying and sterilization with ethylene oxide gas. Part 1. Experimental evaluation of its efficacy and safety. International orthopaedics, 20(3), 142-146.

Langford, R. J., \& Frame, J. W. (2002). Surface analysis of titanium maxillofacial plates and screws retrieved from patients. International journal of oral and maxillofacial surgery, 31(5), 511-518.

Lee, J. S., Fitz Gibbon, E. J., Chen, Y. R., Kim, H. J., Lustig, L. R., Akintoye, S. O., Collins, M. T., \& Kaban, L. B. (2012). Clinical guidelines for the management of craniofacial fibrous dysplasia. Orphanet journal of rare diseases, 7 Suppl 1(Suppl 1), S2.

Meningaud, J. P., Poupon, J., Bertrand, J. C., Chenevier, C., Galliot-Guilley, M., \& Guilbert, F. (2001). Dynamic study about metal release from titanium miniplates in maxillofacial surgery. International journal of oral and maxillofacial surgery, 30(3), 185-188.

Mici, E., \& Belli, E. (2018). Fibrous dysplasia: a complex maxillary reconstruction. The Journal of craniofacial surgery, 29(7), e660-e661.

Pan, Z., \& Patil, P. M. (2014). Titanium osteosynthesis hardware in maxillofacial trauma surgery: to remove or remain? A retrospective study. European jornal of trauma and emergency surgery: oficial publication of theEuropean Trauma Society, 40(5), 587-591.

Pereira, T., Gomes, C. C., Brennan, P. A., Fonseca, F. P., \& Gomez, R. S. (2019). Fibrous dysplasia of the jaws: Integrating molecular pathogenesis with clinical, radiological, and histopathological features. Journal of oral pathology \& medicine: official publication of the International Association of Oral Pathologists and the American Academy of Oral Pathology, 48(1), 3-9.

Pontes-Madruga, T. C., Filgueiras, H., Silva, D., Silva, L., \& Testa, J. (2020). Fibrous dysplasia: rare manifestation in the temporal bone. Brazilian journal of otorhinolaryngology, S1808-8694(20)30099-9. Advance online publication.

Ribeiro, T. A., Coussirat, C., Pagnussato, F., Diesel, C. V., Macedo, F. C., Macedo, C. A., \& Galia, C. R. (2015). Lyophilized xenograft: a case series of histological analysis of biopsies. Cell and tissue banking, 16(2), 227-233. 
Research, Society and Development, v. 10, n. 8, e14910817295, 2021

(CC BY 4.0) | ISSN 2525-3409 | DOI: http://dx.doi.org/10.33448/rsd-v10i8.17295

Sadeghi, S. M., \& Hosseini, S. N. (2011). Spontaneous conversion of fibrous dysplasia into osteosarcoma. The Journal of craniofacial surgery, 22(3), 959961.

Soares, M., Van Dessel, J., Jacobs, R., Yaedú, R., Sant'Ana, E., da Silva Corrêa, D., Madeira, M., Duarte, M., \& Rubira-Bullen, I. (2019). Morphometric evaluation of bone regeneration in segmental mandibular bone defects filled with bovine bone xenografts in a split-mouth rabbit model. International journal of implant dentistry, 5(1), 32 .

Trotta, D. R., Gorny, C., Jr, Zielak, J. C., Gonzaga, C. C., Giovanini, A. F., \& Deliberador, T. M. (2014). Bone repair of critical size defects treated with mussel powder associated or not with bovine bone graft: histologic and histomorphometric study in rat calvaria. Journal of cranio-maxillo-facial surgery: official publication of the European Association for Cranio-Maxillo-Facial Surgery, 42(6), 738-743.

Valentini, V., Cassoni, A., Terenzi, V., Della Monaca, M., Fadda, M. T., Rajabtork Zadeh, O., Raponi, I., Anelli, A., \& Iannetti, G. (2017). Our experience in the surgical management of craniofacial fibrous dysplasia: what has changed in the last 10 years? La nostra esperienza nel trattamento chirurgico della displasia fibrosa cranio-maxillo-facciale: cosa è cambiato negli ultimi 10 anni? Acta otorhinolaryngologica Italica: organo ufficiale della Societa italiana di otorinolaringologia e chirurgia cervico-facciale, 37(5), 436-443.

Verma, A., Jindal, N., Singh, V., \& Sethi, P. (2013). Unusual presentation of monostatic fibrous dysplasia in zygoma. The Journal of craniofacial surgery, 24(6), e592-e594.

Zanettini, L., Vieira, F., Fritscher, G. G., Pagnoncelli, R. M., \& Heitz, C. (2018). Use of Recombinant Human Bone Morphogenetic Protein-2 associated with lyophilized bovine bone in reconstruction of atrophic maxilla. The Journal of craniofacial surgery, 29(7), 1934-1938. 